\title{
Research on Vibration Reduction Characteristics of Continuum and Noncontinuum System on Coupling for High-Power Gear Transmission Based on Particle Damping Materials
}

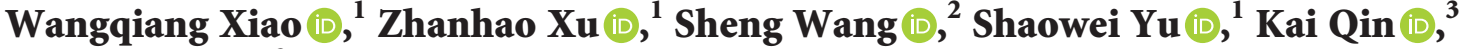 \\ and Dike $\mathrm{Hu} \mathbb{1 D}^{3}$ \\ ${ }^{1}$ School of Aerospace Engineering, Xiamen University, Xiamen, China \\ ${ }^{2}$ Beijing Institute of Electronic System Engineering, Beijing 100854, China \\ ${ }^{3}$ Shanghai Institute of Astronautical Systems Engineering, Shanghai, China \\ Correspondence should be addressed to Wangqiang Xiao; wqxiao@xmu.edu.cn
}

Received 16 June 2020; Accepted 15 June 2021; Published 29 June 2021

Academic Editor: Cristina Castejón

Copyright (C) 2021 Wangqiang Xiao et al. This is an open access article distributed under the Creative Commons Attribution License, which permits unrestricted use, distribution, and reproduction in any medium, provided the original work is properly cited.

High-power gears are widely used in various engineering fields. The gear transmission system is an extremely complex elastic system, which produces complex vibration under internal and external excitation. For the vibration and noise problems caused by transmission error, a discrete element and finite coupling method based on the particle filling rate is proposed. Firstly, the gear dynamic model was established, and the particle damper was installed in the gear to reduce the vibration of the gear. Secondly, through the coupling process, the contact force and contact position between the noncontinuous medium and the continuous medium were correctly transferred to the corresponding nodes of the finite element analysis model. Then, the equivalent displacement mapping of the contact loads' node of the gear was realized, and the transformation of the local coordinate to the global coordinate was carried out. Finally, by combining theoretical analysis with experimental verification, the influence of the filling rate of damping particles on the vibration reduction effect of the gearbox under different working conditions was studied. The $2 \mathrm{~mm}$ tungsten particles were selected, and the particle damper had the best damping effect when the filling rate was $88 \%$.

\section{Introduction}

A gear system is an important part of power transmission and motion in mechanical devices. It has the advantages of large load-carrying capacity, high accuracy, and constant power transmission. Therefore, it is widely used in aviation, ship, automobile, and instrument industries [1]. With the rapid development of science and technology, mechanical equipment is developing towards automation, high strength, large-scale, and high performance. The requirement of high efficiency, strong antivibration, and low noise of the gear mechanism designed and manufactured is gradually increasing [2-5]. Transmission error is the cause of vibration and noise $[6,7]$. The excitation of gear transmission includes the external excitation caused by the prime mover and load as well as the internal excitation caused by time-varying meshing stiffness, time-varying transmission error, and meshing impact [8]. Because of the interaction of internal and external excitation, the dynamic characteristics of the gear transmission system become very complex.

Gearboxes are a kind of equipment mainly composed of structural noise. There are three main transmission paths for vibration and noise: firstly, the meshing sound generated by the gears during the meshing process is transmitted in the form of solid sound through the path of the tooth surface $\longrightarrow$ shaft $\longrightarrow$ bearing $\longrightarrow$ bearing seat $\longrightarrow$ box and is radiated to the outer space of the gearbox through the vibration of each wall surface of the box. The vibration transmission path is shown in Figure 1. Secondly, the meshing sound generated by the gears during the meshing process passes through the internal space of the gearbox $\longrightarrow$ the walls of the box which vibrates to radiate noise to the space outside the box. Thirdly, the meshing sound radiates outward through various slots in gearboxes. About 


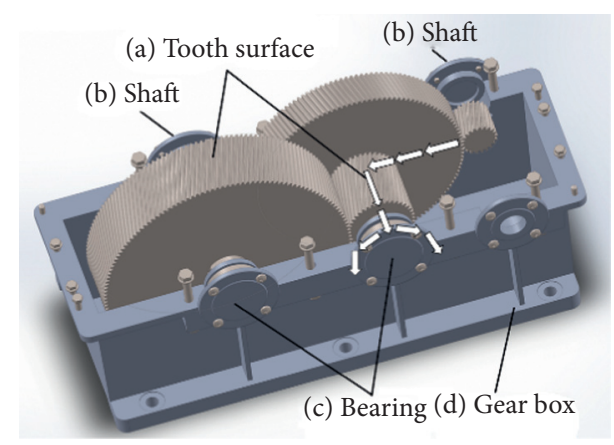

FIgURE 1: Schematic diagram of the first vibration transmission path.

$90 \%-95 \%$ of the radiated sound energy of the gearbox is transmitted through the first way. For the first way, if the vibration is absorbed at the back end of the vibration transmission path, such as the box case, then the effect is poor. If the vibration is absorbed at the front end of the vibration transmission path, the efficiency of vibration reduction is the highest $[9,10]$.

In order to solve the vibration problem caused by exciting force, particle damping technology is introduced into the gear transmission system. The damper is formed by filling particulate matter in the inner cavity of the structure to achieve effective vibration reduction. The lightening hole is located between the tooth surface and the shaft and is at the front end of the transmission path. Particle dampers are installed in the lightening holes to achieve energy dissipation through inelastic collisions and friction between particles and wall of the damper $[11,12]$. And, it can greatly improve the efficiency of vibration reduction. There are many traditional damping measures, and the widely used damping components are rubber isolators [13]. Conventional rubber isolators have many problems: the working temperature range is narrow, and the heat conduction path is blocked, which brings thermal design difficulties. The rubber material is prone to aging, and it needs to be replaced periodically [14]. In order to meet the needs of future product development, it is necessary to develop and design a new vibration reduction technology without interrupting heat transfer path. It is suitable for wide temperature range and wide frequency bandwidth and can avoid introducing linear displacement and angular displacement. Particle damping technology can provide effective broadband vibration reduction in harsh environments such as high and low temperature. It has many advantages, such as remarkable vibration reduction effect, isotropy, high reliability, and no change of the original structure [15-18]. Therefore, this method is very suitable for the harsh working environment of high temperature and oil lubrication in gearboxes.

At present, the study of the energy dissipation mechanism of particle damping technology is mainly applied to the steady-state field [19-21]. Sliding friction and inelastic collisions are the main motions of particles, and they are seldom used in the centrifugal field. Particle damping in the centrifugal field will show different characteristics from that in the steady-state field [22]. When particles are subjected to large centrifugal force, particles will be squeezed to one end far from the center and move close to the wall of the damper [23]. At this time, the movement of particles is dominated by rolling friction and inelastic collisions. When the gear speed is different, the energy dissipation factor of particle damping is obviously nonlinear because of the difference of particle filling rate, particle size, particle density, friction coefficient of the particle surface, recovery coefficient of the particle surface, and installation position of the particle damper.

In previous studies, the energy dissipation mechanism of particles in the centrifugal field of gear transmission caused by friction and collision has been deeply studied [24, 25]. It is proved that adding particles into gears has a certain effect of vibration reduction. Nevertheless, it cannot solve the displacement and stress at any position of the gear system continuum $[26,27]$. When studying the dynamic characteristics of gear systems, the dynamic response can be analyzed by the finite element method. However, after the particle damper is installed in the gear lightening hole, the finite element method cannot solve the discontinuous medium problem because the particle system is a discontinuous medium [28]. The damping matrix [C] of a particle damper in the gear system cannot be directly given in the finite element method. However, the discrete element method can solve all these problems. The basic idea of the discrete element method is to discretize the discontinuous body into independent elements and solve the motion equation of each element by the time step iteration method [29, 30]. For the problem that the frequency response of any gear position cannot be derived from the energy consumption of the particles, this study proposes a method of coupling the discrete element method with the finite element method in the same computational model.

Particle damping is introduced into the dynamic response analysis of gears by using the coupled discrete element-finite element method. It can realize the equivalent mapping of contact load from the discontinuous domain to the gear finite element node $[31,32]$. By analyzing the internal mechanism of particle damping in the gear centrifugal field, the optimum parameters of the particle filling rate are found. Combined with the experiment, the nonlinear dynamic characteristics of gear transmission with particle damping can be calculated and predicted more reasonably.

\section{Discrete Element Model Based on Gear Transmission}

2.1. Gear Meshing Motion Equation. In this study, a pair of cylindrical spur gears with external engagement are analyzed and discussed. The main geometric parameters are shown in Table 1.

As an elastic mechanical system, the gear will produce vibration and noise under the action of dynamic excitation. Therefore, the gear is not only stimulated by external acceleration impact but also by internal excitation caused by stiffness excitation, error excitation, and meshing impact excitation. It causes intense motion of gears. The effect of particle damping on the gear can be used as the additional external damping force of the gear system under the existing 
TABLE 1: Geometric parameters of gear pairs.

\begin{tabular}{|c|c|c|c|c|c|c|}
\hline Gear & Number of teeth & Tooth width $(\mathrm{mm})$ & Normal pressure angle & Module $(\mathrm{mm})$ & Tip clearance coefficient & Materials \\
\hline $\begin{array}{l}\text { Gear } 1 \\
\text { Gear } 2\end{array}$ & $\begin{array}{c}34 \\
107\end{array}$ & $\begin{array}{l}100 \\
100\end{array}$ & 20.0 & 3.5 & 0.25 & $45 \mathrm{Cr}$ \\
\hline
\end{tabular}

boundary conditions. It is equivalent to the additional external load. If the gear system is simplified to the vibration system shown in Figure 2, the nonlinear dynamic equation of the gear can be expressed as follows [33]:

$$
M \ddot{x}+C \dot{x}+(\bar{K}-\Delta K(t))\left(x(t)+x_{s}+e(t)\right)=F(t)+\sum F_{p}(t),
$$

where $M$ is the equivalent mass of the gear pair, $C$ is the damping matrix, $\bar{K}$ represents the average stiffness matrix, $\Delta K(t)$ represents the matrix of the variable stiffness part of the meshing stiffness, $x(t)$ is the vibration displacement vector, $x_{s}$ is the static relative displacement, $e(t)$ is the gear comprehensive transmission error, $F(t)$ represents the external excitation load, and $F_{p}(t)$ is the damping force of the particle system. When particle damping force is used as an external excitation of the gear, its phase can be adjusted. It can reduce the internal excitation, so as to achieve the purpose of vibration reduction.

2.2. Establishment of Discrete Element Model. The particle system is a discrete system, and the gear is a continuous element entity. Both the discrete element method and the finite element method cannot independently and accurately analyze the interaction between the gear systems with particles. The interaction force and displacement between discrete particles and continuous elements can be well solved by the coupling method of finite element and discrete element.

The discrete element method is a numerical method for solving discontinuous media. It can analyze the law of particle motion in the gear discrete system and the effect of interaction between them. The discrete element method used the shell element to mesh by using mesh generation software. The mesh size and density were defined according to the geometric size of the model to ensure the balance between the number of meshes and the quality of meshes. The finite element model after meshing with ANSYS Workbench and discrete element model is shown in Figure 3.

2.3. Discrete Element Calculation of Gear Transmission. In the particle discrete element method, the interaction between particles is regarded as a dynamic process. The contact force and displacement between particles are obtained by tracking the movement of a single particle. The contact force between particles will be produced because of the contact. This group of forces will produce resultant force and resultant moment on the center of gravity of particles. As a

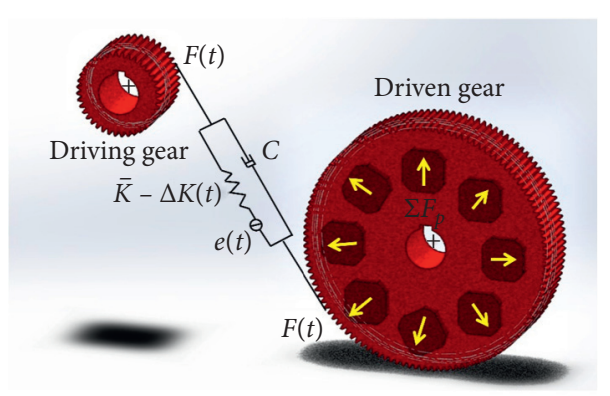

FIGURE 2: Gear transmission vibration system.

result, particles will move. The motion law of particles is calculated in the discrete element method according to Newton's second law.

The motion of a single rigid particle is generated by the resultant force and moment acting on it, which are represented by the translational and rotational motion of the particle. The translational motion of the center of mass is described by its position vector $\vec{x}_{i}$, velocity $\dot{\vec{x}}_{i}$, and acceleration $\ddot{\vec{x}}_{i}$. The rotational motion is described by angular velocity $\vec{\omega}_{i}$ and angular acceleration $\dot{\vec{\omega}}_{i}$. The motion equation consists of two vector equations; one is the translation motion equation related to the resultant force, and the other is the rotation motion equation related to the resultant moment:

$$
\left\{\overrightarrow{F_{i}}=m\left(\ddot{\overrightarrow{x_{i}}}-\overrightarrow{g_{i}}\right), \vec{M}_{i}=I \dot{\overrightarrow{\omega_{i}}}=\left(\frac{2}{5} m R^{2}\right) \dot{\overrightarrow{\omega_{i}}},\right.
$$

where $\overrightarrow{g_{i}}$ is the acceleration of gravity, $I$ represents the principal moment of inertia of the particle, $\vec{F}_{i}$ is the resultant force, $\vec{M}_{i}$ is the combined moment, $m$ is the mass of the particle, and $R$ is the radius of the particle.

In fact, the discrete element method can only affect the particles in direct contact within a single time step by selecting a small enough time step, and the perturbation from any other element cannot be propagated. Therefore, the force acting on each particle is determined only by the particles in direct contact with it. The central finite difference method is used to solve the integral problem in time step $\Delta t$. The translational and rotational accelerations in time $t$ can be expressed as follows:

$$
\left\{\begin{array}{l}
\ddot{\vec{x}}_{i}^{(t)}=\frac{1}{\Delta t}\left(\dot{\vec{x}}_{i}^{(t+(\Delta t / 2))}-\dot{\vec{x}}_{i}^{(t-(\Delta t / 2))}\right), \\
\dot{\vec{\omega}}_{i}^{(t)}=\frac{1}{\Delta t}\left(\dot{\vec{\omega}}_{i}^{(t+(\Delta t / 2))}-\dot{\vec{\omega}}_{i}^{(t-(\Delta t / 2))}\right) .
\end{array}\right.
$$




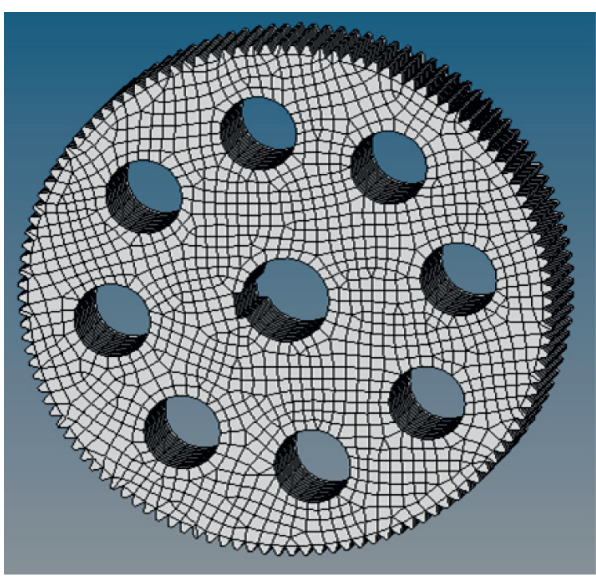

(a)

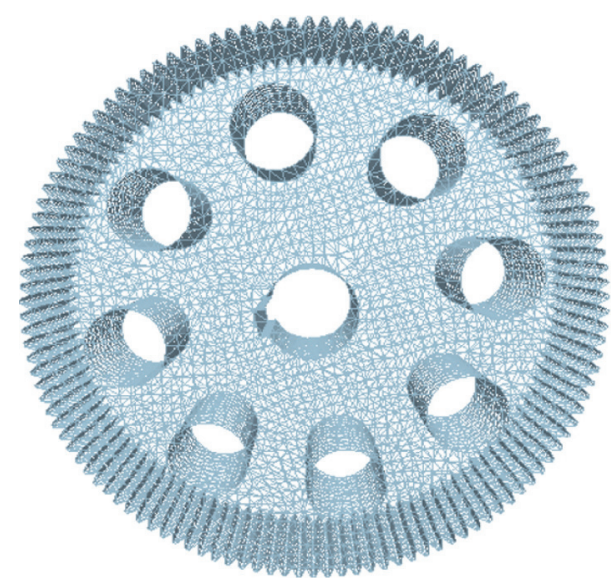

(b)

Figure 3: Gear transmission model. (a) Finite element model. (b) Discrete element model.

The speed value at time $t+(\Delta t / 2)$ is

$$
\left\{\begin{array}{l}
\dot{\vec{x}}_{i}^{(t+(\Delta t / 2))}=\dot{\vec{x}}_{i}^{(t-(\Delta t / 2))}+\left(\frac{\vec{F}_{i}^{(t)}}{m}+\vec{g}_{i}\right) \Delta t, \\
\vec{\omega}_{i}^{(t+(\Delta t / 2))}=\vec{\omega}_{i}^{(t-(\Delta t / 2))}+\left(\frac{\vec{M}_{i}^{(t)}}{I}\right) \Delta t .
\end{array}\right.
$$

The displacement at time $(t+\Delta t)$ can be obtained from the speed at time $t+(\Delta t / 2)$ :

$$
\begin{aligned}
& \vec{x}_{i}^{(t+\Delta t)}=\vec{x}_{i}^{(t)}+\dot{\vec{x}}_{i}^{(t+(\Delta t / 2))} \Delta t, \\
& \vec{\omega}_{i}^{(t+\Delta t)}=\vec{\omega}_{i}^{(t)}+\dot{\vec{\omega}}_{i}^{(t+(\Delta t / 2))} \Delta t .
\end{aligned}
$$

Aftertime, the particle element reaches a new position and generates new contact force and moment. Thus, new acceleration and angular acceleration are generated, and the cycle is continuously traversed. The force acting on the particle unit at any time depends only on the particle itself and other particles in direct contact with it.

2.4. Discrete Element System of Particles. The tangential force of friction and normal force is produced by contact collision between particles. Based on the accuracy and speed of calculation, elastic force and damping force are introduced, and the contact force between particles is decomposed into tangential force and normal force. The normal direction is simplified to a linear contact model, and the tangential direction is simplified to a Coulomb friction model. A particle damper is installed on the gear system, and the contact model between particles and between particles and damping wall is given. As shown in Figure $4, k_{n}$ is the normal stiffness of particles, $c_{n}$ represents the normal damping of particles, $k_{t}$ is the tangential stiffness of particles, and $c_{t}$ represents the tangential damping of particles.

During the motion of the gear system, the motion equation of particles in the damper at a certain time is as follows:

$$
\begin{aligned}
\sum_{j=1}^{s_{i}}\left(F_{n}+F\right) & =m_{i} \ddot{p}_{i}-m_{i} g-m_{i} \omega^{2} R, \\
\sum_{j=1}^{s_{i}} T & =I_{i} \frac{\mathrm{d}^{2} \varphi_{i}}{\mathrm{~d} t^{2}} .
\end{aligned}
$$

In the above equation, $F_{n}$ and $F_{t}$ are the normal contact force and tangential contact force between particles $i$ and $j$, $P_{i}$ represents the particle displacement vector, $g$ is the gravitational acceleration, $m_{i}$ is the mass of the particle, $\omega$ is the angular velocity of particle $i, R$ is the displacement of particle $i$ to the gear rotating shaft, $i$ is the particle moment of inertia, $\varphi_{i}$ represents the particle angular displacement vector, $T$ is the torque generated in the tangential direction, and $S_{i}$ is the number of particles in contact with particle $i$ at a certain time.

The normal force can be expressed as

$$
\begin{aligned}
F_{n} & =F_{k n}+F_{c n}, \\
& =-k_{n} D_{n}-c_{n} V_{n},
\end{aligned}
$$

where $D_{n}$ is the amount of normal deformation between particles and $V_{n}$ represents the normal relative velocity between particles, which can be derived from Hertz contact theory.

$P-P$ is used to represent particles and particles, and $P-W$ is used to represent particles and damper walls. The normal elastic coefficient $k_{n}$ can be expressed as 


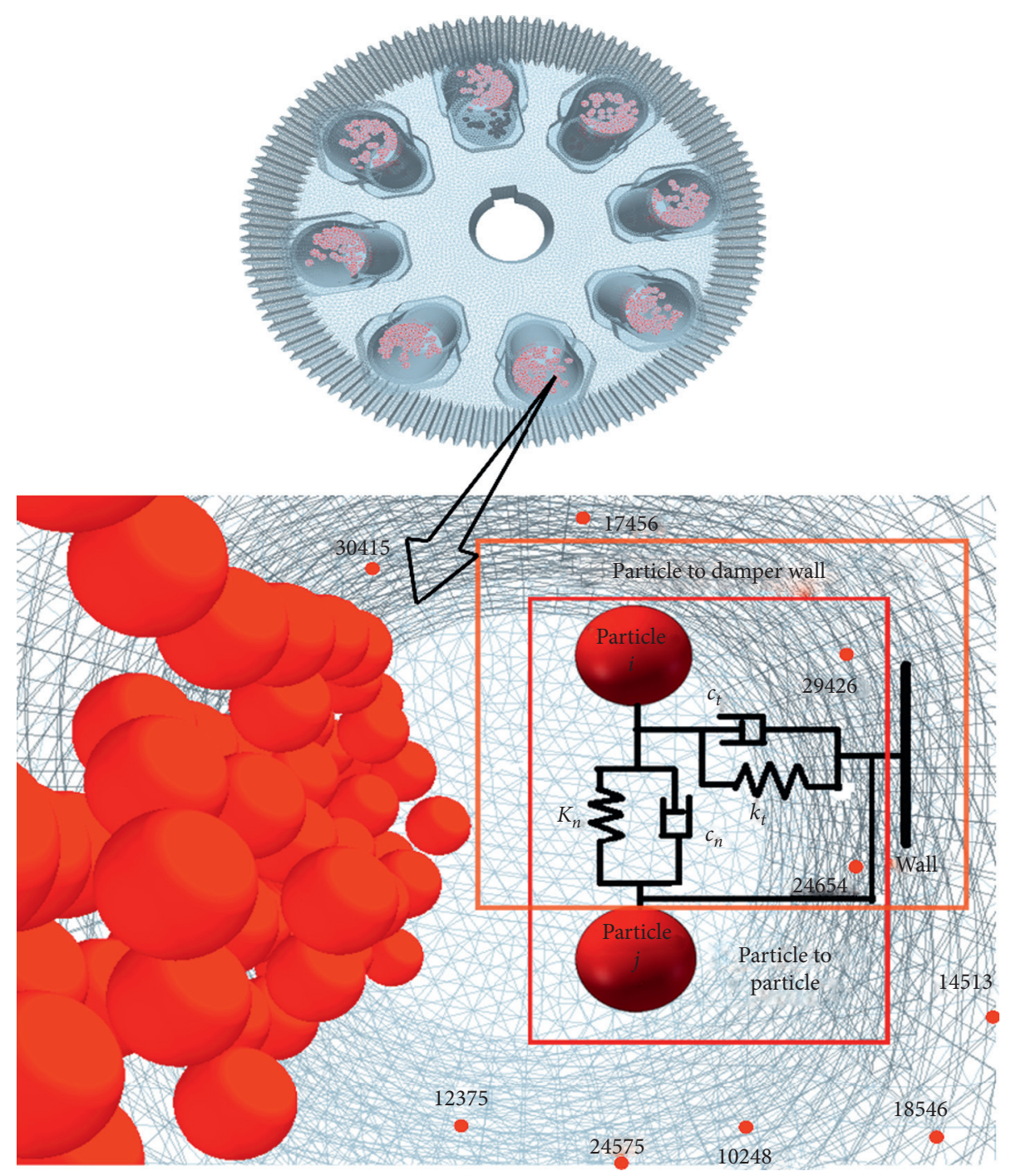

FIgURE 4: Gear model equipped with particle dampers.

$$
\begin{cases}k_{n}=\frac{2}{3}\left(\frac{1-V_{i}^{2}}{E_{i}}\right)^{-1}\left(\frac{2}{R_{i}}\right)^{-(1 / 2)}, & P-P, \\ k_{n}=\frac{4}{3}\left(\frac{1-V_{i}^{2}}{E_{i}}+\frac{1-V_{j}^{2}}{E_{j}}\right)^{-1}\left(\frac{1}{R_{i}}\right)^{-(1 / 2)}, & P-W, \\ c_{n}=2 \sqrt{m k_{n}}, & \end{cases}
$$

where $E$ represents the elastic modulus of the contact element, $V$ is Poisson's ratio, $R$ is the particle radius, and $m$ is the equivalent mass of the contact unit.

The tangential force can be expressed as

$$
F_{t}=F_{k t}+F_{c t}=-k_{t} D_{t}-c_{t} V_{t},
$$

where $D_{t}$ is the amount of tangential deformation between particles and $V_{t}$ is the tangential relative velocity between particles. According to the Hertz contact model, $k_{t}$ and $c_{t}$ can be derived as

$$
k_{t}=r k_{n}
$$

where $r$ is the proportional coefficient:

$$
\begin{array}{ll}
r=\frac{1-V_{i}}{1-0.5 V_{i}}, & P-P, \\
r=\frac{\left(\left(1-V_{i}\right) / G_{i}\right)+\left(\left(1-V_{j}\right) / G_{j}\right)}{\left(\left(1-0.5 V_{i}\right) / G_{i}\right)+\left(\left(1-0.5 V_{j}\right) / G_{j}\right)}, & P-W, \\
c_{t}=2 \sqrt{m k_{t}}, &
\end{array}
$$

where $m$ is the equivalent mass of the contact element and $G$ is the shear modulus of the contact unit. 


\section{Discrete Element and Finite Element Coupling Simulation}

In the process of gear coupling analysis, the coupling process between discrete element and finite element is mainly to transfer the mechanical parameters between discrete element and finite element. The contact force and position between the bulk medium and continuous medium can be correctly transferred to the corresponding nodes in the finite element analysis model.

For the gear finite element and discrete element coupling calculation method, the discrete element method is used to calculate the excitation state of the particle system under the gear excitation, and then, the damping force of the particle system to the structure can be calculated. The damping force is transformed into the gear finite element and repeated for several time steps to obtain the whole simulation time particle damping force. Discrete element can only recognize triangular shell meshes. In order to achieve coupling, the element numbers of the model at the same position in the discrete element and the finite element must correspond one by one. In practical analysis, some methods are needed to transfer the contact force from the discrete element region to the finite element region. The overall process is shown in Figure 5.

The path of force transmission of the gear system is tooth surface-lightening hole-center shaft. The excitation is generated on the tooth surface and transmitted rapidly from the gear center shaft. On the transmission path, the vibration is transmitted from the tooth surface to the particle system in the lightening hole. The particle system is stimulated, and it vibrates and generates the opposite damping force, which reduces the transmission of vibration. The discrete element method is used to calculate the effect of the particle system on the gear lightening hole, and the damping force of the particle system on the gear can be calculated based on the ID number of discrete element. Figure 6 is the force chain system of the particle system and the gear lightening hole. The red part is the force chain between the particle system and the lightening hole, which is the most intuitive expression of particle damping effect.

The contact effect between the damper particle and the triangular shell element on the outer wall of the damper is shown in Figure 7. It can be seen that there are collisions of single or multiple particles on each triangular shell element. Based on the element ID number, particle damping force on each element ID is synthesized in the local coordinate system, and the force on each shell element is calculated, as shown in Table 2. In order to be coupled with the finite element method, it is necessary to convert the damping force into nodal load. Based on the shape function method, the load acting on the surface element is converted to the nodal force of the triangular element. The force of the transformed node is regarded as the boundary load of the dynamics of the gear system. Finally, the effect of the particle damper on gears is characterized.

The transformation method of the shape function is as follows. Figure 8 is a tetrahedral element with particle $M$

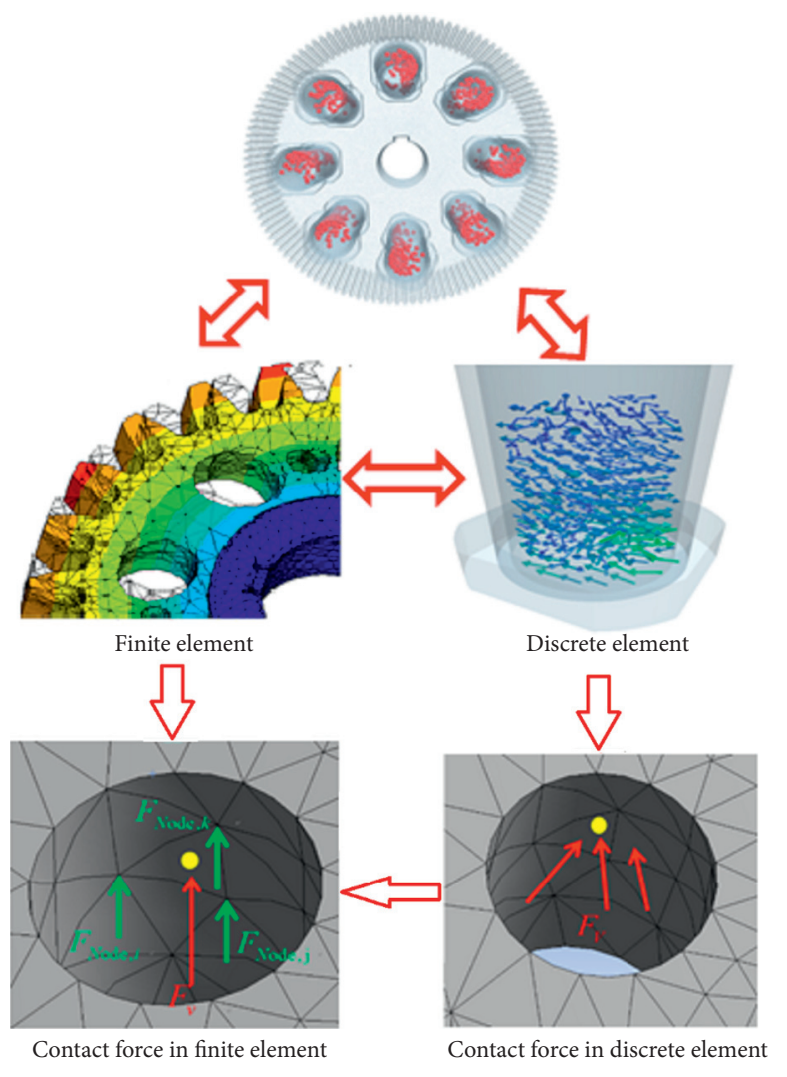

FIgURE 5: Discrete element and finite coupling method total flow of gear transmission.

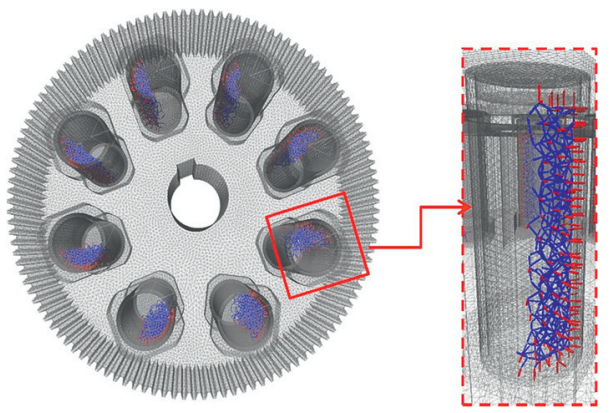

FIGURE 6: Force chain transfer in gear-particle transmission system.

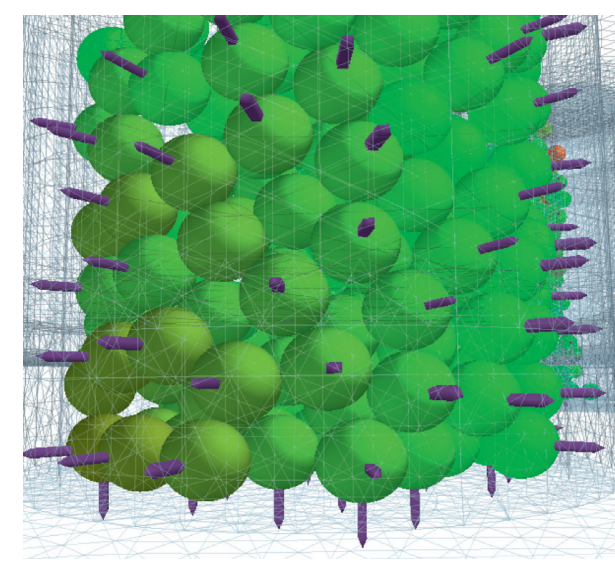

Figure 7: Force diagram of the shell element. 
TABLE 2: Force of collision between particles and shell elements.

\begin{tabular}{|c|c|c|c|c|c|c|c|c|c|}
\hline \multirow{2}{*}{ Unit ID } & \multicolumn{3}{|c|}{ Position coordinates } & \multicolumn{3}{|c|}{ Normal force $(\mathrm{N})$} & \multicolumn{3}{|c|}{ Tangential force $(\mathrm{N})$} \\
\hline & $X$ & $Y$ & $Z$ & $X$ & $Y$ & $Z$ & $X$ & $Y$ & $Z$ \\
\hline 11 & 66.907 & -41.132 & -3.088 & -0.400 & -0.034 & 0.000 & 0.008 & -0.095 & 0.136 \\
\hline 62 & -83.172 & -32.030 & 30.498 & -0.977 & 0.886 & 0.000 & 0.395 & 0.436 & -0.205 \\
\hline 108 & 3.984 & -94.519 & -21.003 & -0.008 & 0.159 & 0.000 & -0.060 & -0.003 & -0.013 \\
\hline 169 & 10.902 & 60.452 & -1.612 & 0.200 & 0.094 & 0.000 & -0.033 & 0.069 & -0.003 \\
\hline 211 & 53.436 & -28.097 & 29.801 & -0.064 & -1.327 & 0.000 & 0.464 & -0.022 & -0.273 \\
\hline 314 & 47.429 & 24.081 & -11.863 & 0.203 & -0.104 & 0.000 & 0.020 & 0.039 & -0.049 \\
\hline 320 & -47.329 & 85.443 & -33.412 & 0.075 & -0.068 & 0.000 & 0.029 & 0.033 & 0.005 \\
\hline 347 & 66.918 & -40.405 & -33.501 & -0.149 & -0.032 & 0.000 & 0.012 & -0.053 & 0.039 \\
\hline 427 & 60.966 & -37.472 & -35.500 & 0.000 & 0.000 & 0.447 & -0.181 & -0.050 & 0.000 \\
\hline 472 & -46.827 & 85.938 & -20.327 & 0.099 & -0.089 & 0.000 & 0.011 & 0.013 & 0.054 \\
\hline 476 & 8.472 & -93.577 & -19.694 & -0.173 & 0.537 & 0.000 & -0.055 & -0.018 & 0.113 \\
\hline 603 & 64.432 & -34.583 & -5.804 & -0.346 & -0.240 & 0.000 & 0.101 & -0.146 & 0.035 \\
\hline 728 & -47.045 & 65.533 & 22.849 & 1.155 & 0.994 & 0.000 & -0.367 & 0.426 & 0.218 \\
\hline 769 & 12.012 & 58.407 & -13.387 & 0.688 & 0.444 & 0.000 & -0.078 & 0.121 & 0.295 \\
\hline 802 & 75.985 & 18.612 & -33.287 & -0.284 & -0.014 & 0.000 & 0.003 & -0.051 & 0.096 \\
\hline 804 & 18.303 & -82.488 & -13.134 & -1.296 & 0.265 & 0.000 & -0.050 & -0.247 & 0.528 \\
\hline 879 & -86.747 & 42.234 & -35.500 & 0.000 & 0.000 & 0.044 & -0.019 & 0.004 & 0.000 \\
\hline 1054 & -100.608 & -34.893 & -20.538 & 1.135 & 2.208 & 0.000 & -0.843 & 0.434 & 0.106 \\
\hline$\ldots$ & $\ldots$ & $\ldots$ & $\ldots$ & $\ldots$ & $\ldots$ & $\ldots$ & $\ldots$ & $\ldots$ & $\ldots$ \\
\hline
\end{tabular}

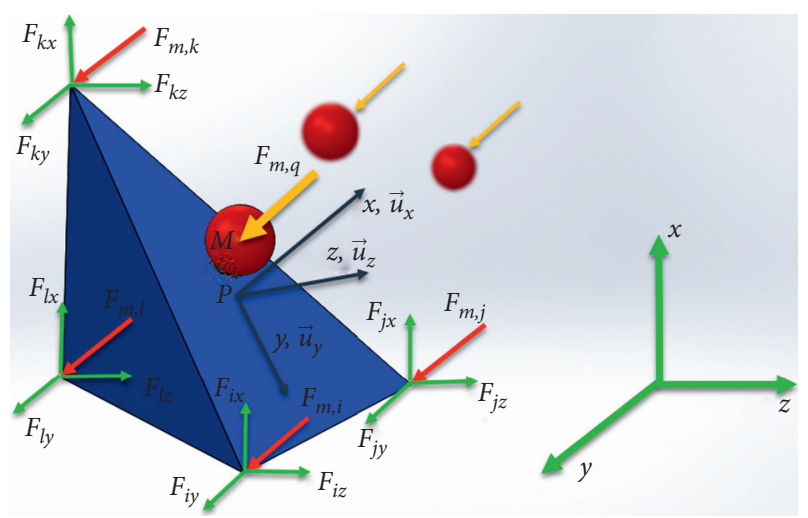

Figure 8: Contact force conversion diagram of tetrahedral elements.

acting on a triangular plane element. The graph shows a local coordinate system $(x, y, z)$ located at the center of $P$ point in the $x-y$ plane, which is consistent with the plane of the triangular element. $u_{i j}$ is the unit vector along point $i$ and point $j$, and $u_{i k}$ is the unit vector along point $i$ and point $k$. The unit normal vector $n$ of a plane triangular element is

$$
\bar{n}=\frac{\overrightarrow{u_{i j}} \times \overrightarrow{u_{i k}}}{\left|\overrightarrow{u_{i j}} \times \overrightarrow{u_{i k}}\right|} .
$$

The $x$ direction of the local coordinate system is the same as $u_{i j} . \overrightarrow{u_{x}}, \overrightarrow{u_{y}}$, and $\overrightarrow{u_{z}}$ of the local coordinate system can be expressed as

$$
\begin{aligned}
& \overrightarrow{u_{x}}=\overrightarrow{u_{i j}}, \\
& \overrightarrow{u_{y}}=\vec{n} \times \overrightarrow{u_{x}}, \\
& \overrightarrow{u_{z}}=\vec{n} .
\end{aligned}
$$

The relationship between the local coordinate system and global coordinate system is as follows:

$$
\begin{aligned}
\{x, y, z\}^{T} & =\left[T_{t}, 1\right]\{X, Y, Z\}^{T}, \\
{\left[T_{t}, 1\right] } & =\left[\left\{\overrightarrow{u_{x}}\right\}\left\{\overrightarrow{u_{y}}\right\}\left\{\overrightarrow{u_{z}}\right\}\right]^{T} .
\end{aligned}
$$

For the bending response of the model shell and the plate surface, each node has six degrees of freedom, including three displacements and three angles. When the bending moment is much larger than the in-plane torque, $\theta_{z}$ can be neglected. The displacement matrix $\left\{U_{6 \times 1}\right\}$ can be established by the shape function $[N]_{6 \times 18}$ and the node displacement $\{S\}_{18 \times 1}$ :

$$
\{U\}_{6 \times 1}=[N]_{6 \times 18}\{S\}_{18 \times 1},
$$

where $\{U\}^{T}=\left\{u_{x}, u_{y}, u_{z}, \theta_{x}, \theta_{y}, \theta_{z}\right\}$ :

$$
\begin{aligned}
\{S\}^{T}= & \left\{u_{x i}, u_{y i}, u_{z i}, \theta_{x i}, \theta_{z i} \ldots \ldots \ldots \ldots \ldots \ldots u_{x k}, u_{y k}, u_{z k},\right. \\
& \left.\theta_{x k}, \theta_{z k}\right\} .
\end{aligned}
$$

The external virtual work induced by contact force is as follows:

$$
\begin{aligned}
\delta W & =\sum_{m=1}^{M}\left\{\delta U_{m}\right\}_{1 \times 6}^{T}\left\{\overrightarrow{F_{c, m}}\right\}_{6 \times 1}, \\
& =\{\delta S\}_{1 \times 18}^{T} \sum_{m=1}^{M}\left\{N_{m}\right\}_{18 \times 6}^{T}\left\{\overrightarrow{F_{c, m}}\right\}_{6 \times 1},
\end{aligned}
$$

where $\left\{\overrightarrow{F_{c, m}}\right\}_{6 \times 1}$ is the contact force vector acting on the contact point $m$ in the local coordinate system and $M$ is the number of contact points at which the particles act on this planar triangular element. The equivalent nodal force in the local coordinate system is as follows: 


$$
\left\{\vec{F}_{c, n o}\right\}_{18 \times 1}=\sum_{m=1}^{M}\left\{N_{m}\right\}_{18 \times 6}^{T}\left\{\overrightarrow{F_{c, m}}\right\}_{6 \times 1}
$$

The contact force in the local coordinate system is transformed into the contact force in the global coordinate system:

$$
\left\{\vec{F}_{c, m}\right\}_{6 \times 1}=\left[T_{t, 2}\right]_{6 \times 6}\left\{F_{c, m}\right\}_{6 \times 1},
$$

where

$$
\left[T_{t, 2}\right]_{6 \times 6}=\left[\begin{array}{cc}
T_{t, 1} & 0 \\
0 & T_{t, 1}
\end{array}\right]
$$

The nodal forces in the local coordinate system are transformed into those in the global coordinate system:

$$
\left\{\vec{F}_{c, n o}\right\}_{18 \times 1}=\left[T_{t, 2}\right]_{18 \times 18}^{T}\left\{\vec{F}_{c, n o}\right\}_{18 \times 1},
$$

where

$$
\left[T_{t, 3}\right]_{18 \times 18}=\left[\begin{array}{ccc}
T_{t, 2} & 0 & 0 \\
0 & T_{t, 2} & 0 \\
0 & 0 & T_{t, 2}
\end{array}\right] .
$$

Substituting (19) and (21) into (18), finally, the contact force formula of the nodes in the global coordinate system is obtained as follows:

$$
\left\{\vec{F}_{c, n o}\right\}_{18 \times 1}=\sum_{m=1}^{M}\left[T_{t, 3}\right]_{18 \times 18}^{T}\left\{N_{m}\right\}_{18 \times 6}^{T}\left[T_{t, 2}\right]_{6 \times 6}^{T}\left\{F_{c, m}\right\}_{6 \times 1}
$$

The forces acting on boundary particles and tetrahedron elements in Table 2 are converted to corresponding node labels. Finally, the damping force of particles to the structure can be obtained, as shown in Table 3 .

\section{Effect of Particle Damping Parameters}

4.1. Design of Damper. Particle dampers are highly nonlinear dampers, and the damping mechanism varies with the filling rate, material, and size of particles. Based on the premise of variable parameters, the design of the damper is particularly important. The larger the size of the damper, the more particles can be filled, and the damping effect will be improved accordingly. Because the structure of the gearbox will limit the size of damper, the maximum utilization of effective space should be considered in the selection of damper shape. Strive to obtain the best quality ratio to get the best use area.

Based on the structure of the gearbox and gear, this study designs a special damper, which can be easily installed and disassembled. A cylindrical cavity damper with a square lid was designed. The diameter of the circular surface was $70 \mathrm{~mm}$. The length of the cylinder was $130 \mathrm{~mm}$, and the material of the damper was $\mathrm{ZAlZn6Mg}$ aluminum alloy. The two cylindrical ends were closely fitted with the side of the gear. The middle part was fitted with the interference of the
TABLE 3: Conversion between element force and node force.

\begin{tabular}{lccc}
\hline \multirow{2}{*}{ Element number } & \multicolumn{3}{c}{ Element load } \\
\hline 2 & 0.257 & $Y$ & $Z$ \\
7 & 0.000 & -0.232 & -11.373 \\
8 & 0.000 & -0.589 & -1.134 \\
11 & 0.000 & -4.956 & -1.812 \\
14 & -1.372 & -0.671 & 0.000 \\
17 & -4.773 & 0.008 & 0.000 \\
20 & -4.389 & 9.027 & -9.418 \\
21 & -0.416 & -26.976 & 0.000 \\
27 & -8.466 & -8.833 & -9.732 \\
36 & -0.194 & 0.000 & -0.533 \\
40 & -2.694 & 0.000 & -11.198 \\
54 & -6.364 & 0.000 & -8.608 \\
55 & 3.324 & 0.000 & -6.595 \\
56 & 0.190 & 0.000 & -9.014 \\
69 & -2.841 & 6.833 & -8.843 \\
121 & 0.000 & -0.004 & 0.000 \\
343 & 0.257 & -0.232 & -11.373 \\
& & &...
\end{tabular}

lightening hole of the gear to ensure the axial fixing constraint of the damper, as shown in Figure 9.

In the finite element analysis, the vibration response of the gear system is solved by the mode superposition method. Damping particles with different parameters are filled in the lightening hole of the gear system, and dynamic loads caused by stiffness excitation, error excitation, and impact excitation are considered. Finally, the time-domain response of any point on the gear system is calculated, and the frequency-domain response of each point can be obtained by Fourier transform. The curves of displacement, velocity, and acceleration at each point can be obtained.

\subsection{Particle Damping Effect at Different Filling Rates at Variable Speed}

4.2.1. Rough Optimization of Filling Rate. The volume filling rate of particles directly affects the mass of individual dampers and arrangement of the internal particles, thereby affecting the energy consumption effect. The 100\% filling rate is expressed as the mass of fully filled particles in the damper. The filling rate of particles is an important factor affecting the damping effect of particles. In this paper, the filling rate of particles is taken as the key research object.

Under different rotational speeds, different filling rates of damping particles have different damping effects on gears. The parameters such as particle size and particle material in each damper are fixed. When the filling space of the damper was limited, the particles with larger proportion were preferred. Therefore, tungsten alloy was used as the material in this study. Poisson's ratio was 0.28 , density was $1.935 * 10^{4} \mathrm{~kg} / \mathrm{m}^{3}$, and elastic modulus was $3.24 * 10^{11} \mathrm{~Pa}$. In this study, the particle size of tungsten with a diameter of $2 \mathrm{~mm}$ was taken as an example, and the filling rate was $60 \%$, $70 \%, 80 \%, 85 \%, 90 \%$, and $95 \%$, and $2 \mathrm{~mm}$ equal diameter particles were selected for the comparative study. Figure 10 showed the filling effect of damped particles filled with 


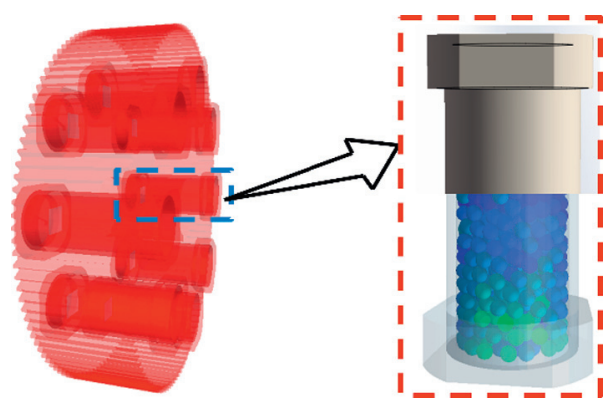

Figure 9: Damper model.

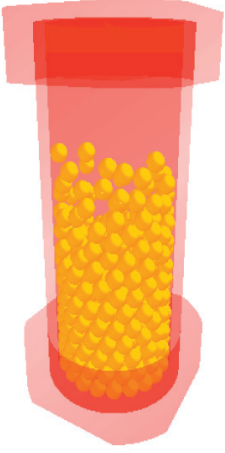

(a)

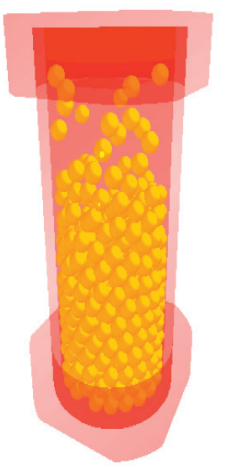

(b)

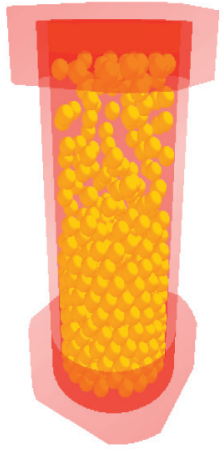

(c)

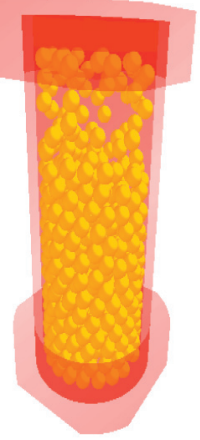

(d)

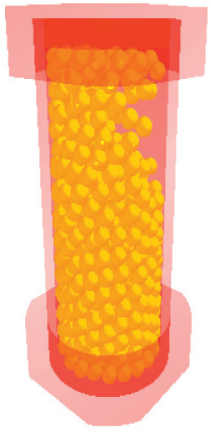

(e)

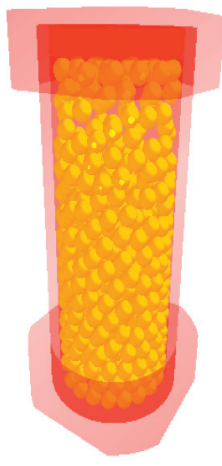

(f)

Figure 10: Effect of different particle filling rates. (a) 60\%. (b) $70 \%$. (c) $80 \%$. (d) $85 \%$. (e) $90 \%$. (f) $95 \%$.

different filling rates in the damper. Damping particles need to be able to travel in a limited space to increase the chance of friction and collision between particles.

In order to study the effect of particle vibration reduction with different filling rates, the acceleration response of the gear center axis position was selected as the basis for evaluation. The response curves of the structure at different rotational speeds and particle filling rates were calculated. The transmission ratio was 3 . When the input of the driving wheel was $300 \mathrm{rpm}$, the driven wheel was $100 \mathrm{rpm}$. Figure 11 shows the acceleration spectra of different particle filling rates at different frequencies. It could be seen that the acceleration value of $90 \%$ particle filling rate was the smallest, so the damping effect is the best.

Different gear speeds and particle filling rates were set, respectively, and the acceleration response of particles was analyzed. The damping particles need enough space for friction and collision. In the range of $0-1000 \mathrm{rpm}$ speed, with the increase of particle speed, the particle damping effect was more obvious. With the increase of rotational speed, the centrifugal force increased gradually. The particles were attached to the wall of the damper far away from the center by the centrifugal force. The gap between the particles also gradually became smaller. With the increase of the gear centrifugal field, when the rotational speed exceeds a value, the particle damping effect decreases sharply due to the effect of strong centrifugal force. The acceleration response diagram of different particle filling rates at different speeds is shown in Figure 12.
4.2.2. Detailed Optimization of Filling Rate. According to the preliminary analysis of the particle filling rate, $90 \%$ filling rate has better damping effect. Therefore, the damping effect of the fine filling rate is further explored with $90 \%$ filling rate as the boundary. The filling rate is $87 \%, 88 \%, 89 \%, 90 \%, 91 \%$, and $92 \%$, and the particle size is $2 \mathrm{~mm}$. As shown in Figure 13, the acceleration response value of $88 \%$ filling rate is the smallest among the acceleration response values of different particle filling rates at different rotational speeds. Therefore, $88 \%$ is the best value for fine filling rate optimization.

\section{Experimental Verification for Gear Transmission System}

5.1. Experimental Bench for Gear Transmission System. The effect of the particle filling rate on vibration reduction of the gearbox at different rotational speeds was studied by the particle damper test. The test device includes gear box, acceleration sensor, data acquisition instrument, and controller. The experimental device and its schematic diagram are shown in Figures 14 and 15, respectively. The gear test box was controlled by the main control panel. The speed sensor was arranged on the gear bearing seat to measure the vibration of the gear box. The particle damper is installed in the gear lightening hole.

Figure 15 shows the main devices for a particle damping test of a gear system. In order to measure the vibration of the running gear, a 3-directional wireless acceleration sensor was installed at the bearing cap closest to the test gear. This 


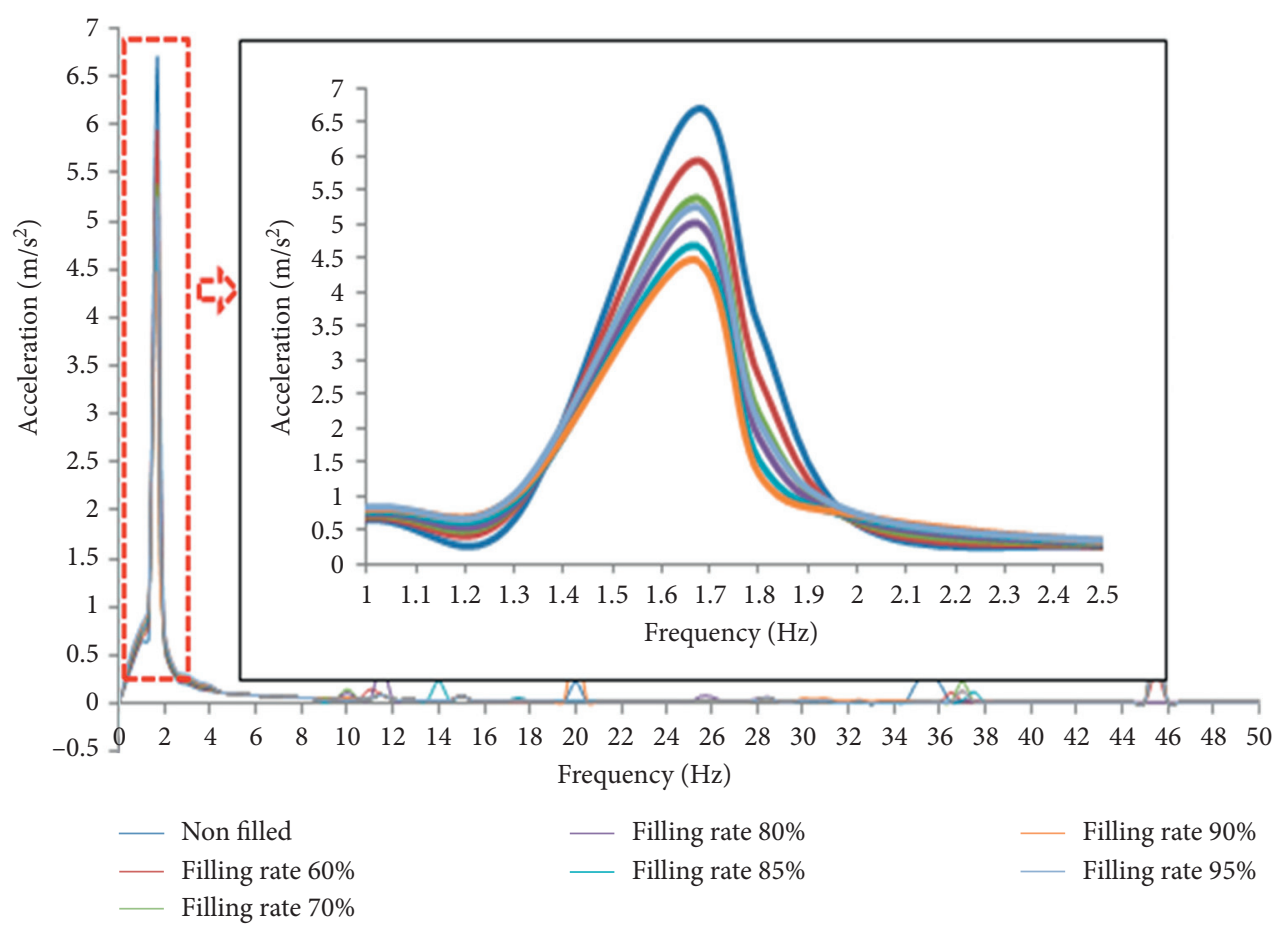

FIGURE 11: Acceleration spectrum.

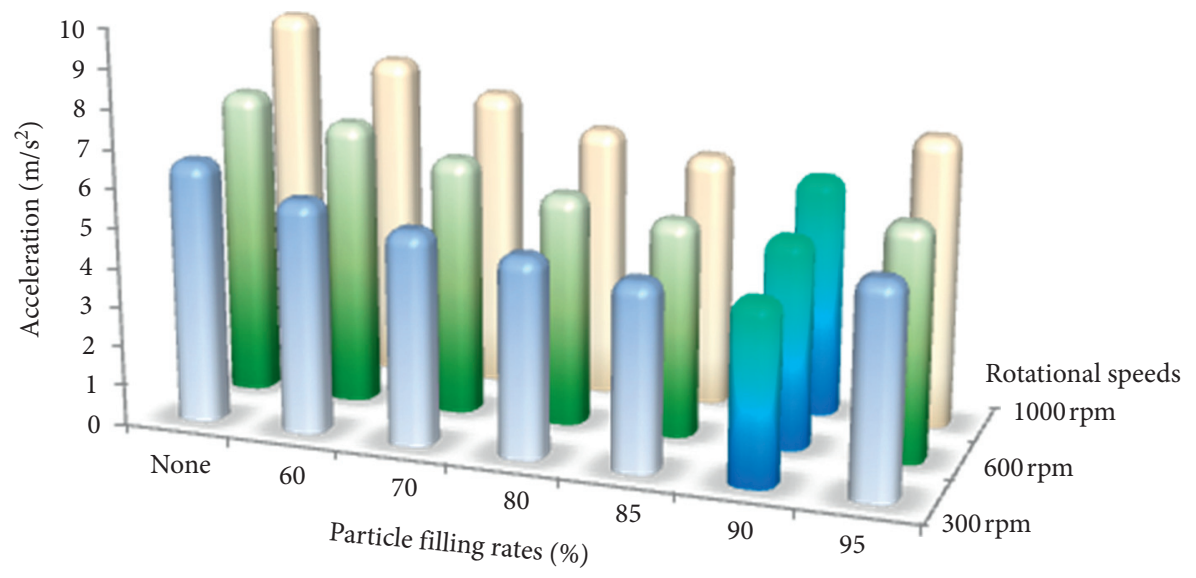

FIGURE 12: Acceleration response of different particle filling rates at different speeds.

sensor can collect the acceleration signals of $X, Y$, and $Z$ directions simultaneously. After a signal has been collected, it is transmitted to the data-collecting instrument. Figure 16 is an image of the device.

5.2. Validation of Particle Vibration Reduction Effect. The experimental data with different filling rates were selected to analyze, and the effect of different filling rates on vibration reduction was explored. Table 4 shows the root mean square acceleration of particles with different filling rates at gear speeds of $300 \mathrm{rpm}, 600 \mathrm{rpm}$, and $1000 \mathrm{rpm}$, respectively. When the filling rate was $88 \%$, the measured acceleration RMS value was the smallest.
Compared with none particles, the vibration acceleration spectra at the filling rate of $88 \%$ are shown in Figure 17. When the speed was $300 \mathrm{rpm}$, the red line was the response curve of the gear bearing seat with none particles and the green line was the response curve of the structure after adding $2 \mathrm{~mm}$ tungsten particles. The response curves of the structure before and after adding the particle damper were quite different, and the peaks at the relevant frequencies were obvious. This showed that the vibration amplitude of the structure decreases obviously due to the effect of particle damping after adding particle damping.

As shown in Figure 18, the solid line indicates the simulated value and the dashed line indicates the test value. It is a comparison between simulation and 


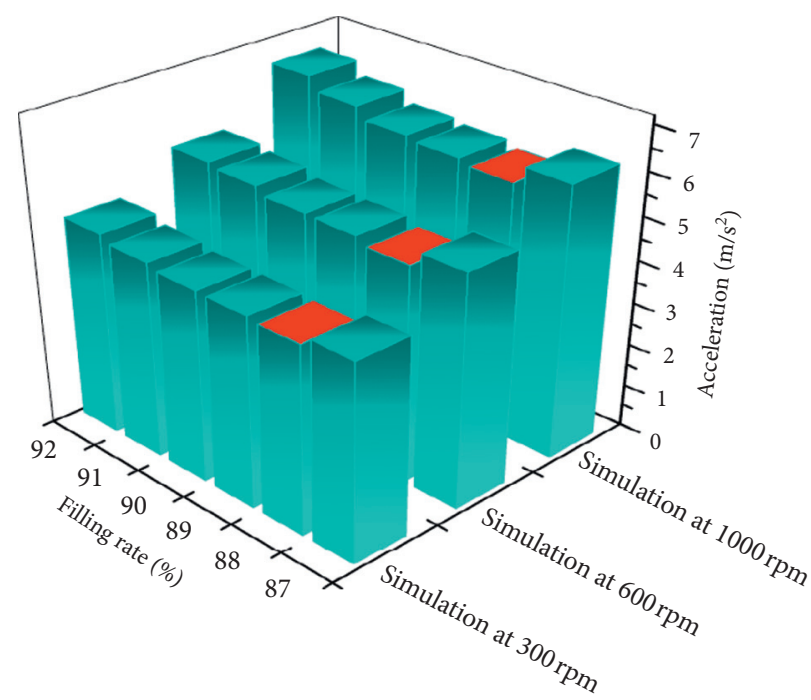

FIGURE 13: Effect of refining the filling rate.

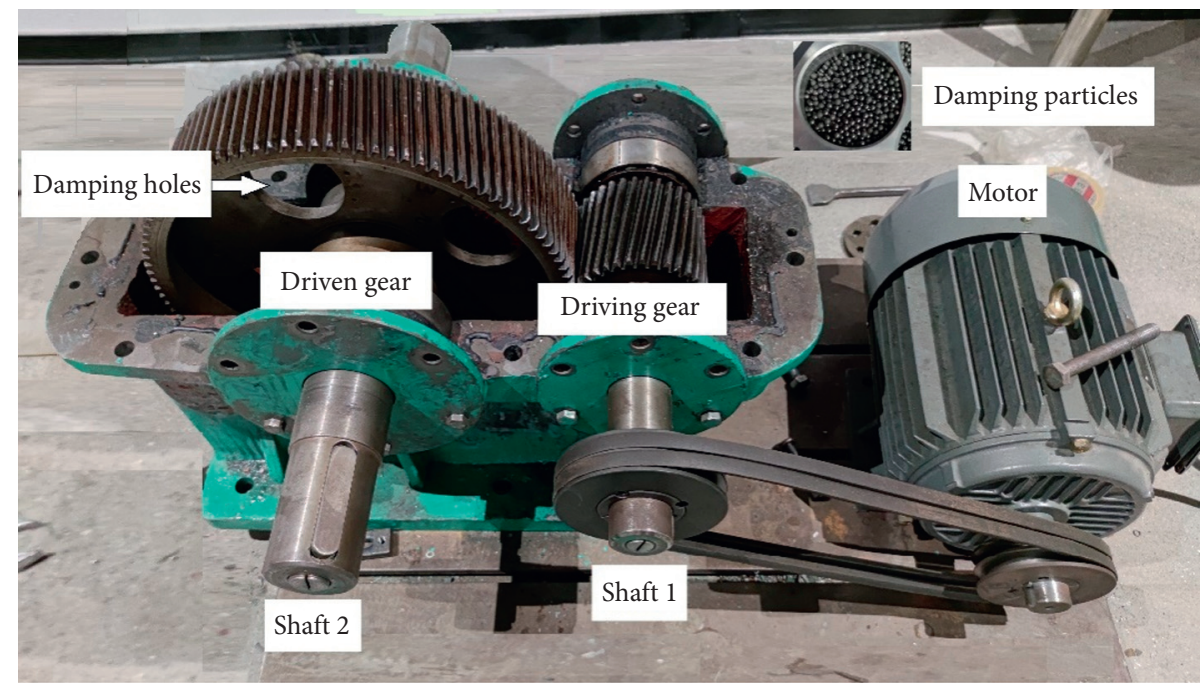

FIGURE 14: Experimental rig diagram.

experimental results, which were highly similar. This was consistent with the theoretical calculation, which showed the correctness of the theoretical calculation. The damping particles need to ensure a certain movement stroke in a limited space, so as to increase the chance of friction and collision between particles and particles. Particles with different filling rates had different energy dissipation effects. Therefore, the choice of fill rate is particularly important. The right filling rate has the best damping effect. In this study, when the filling rate of $2 \mathrm{~mm}$ tungsten particles is $88 \%$, the damping effect of the particle damper is the best. 


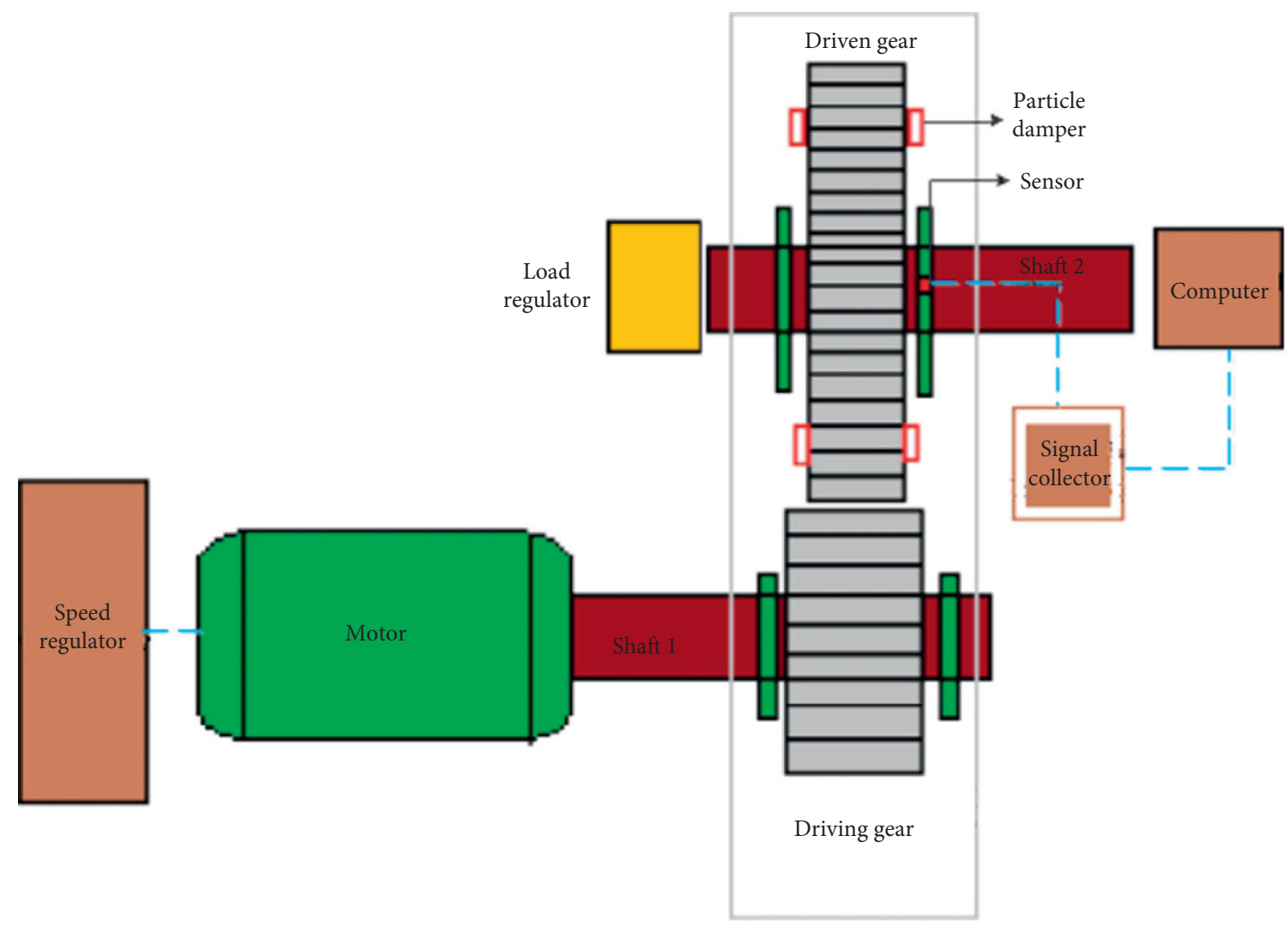

FIGURE 15: Schematic of the test system.
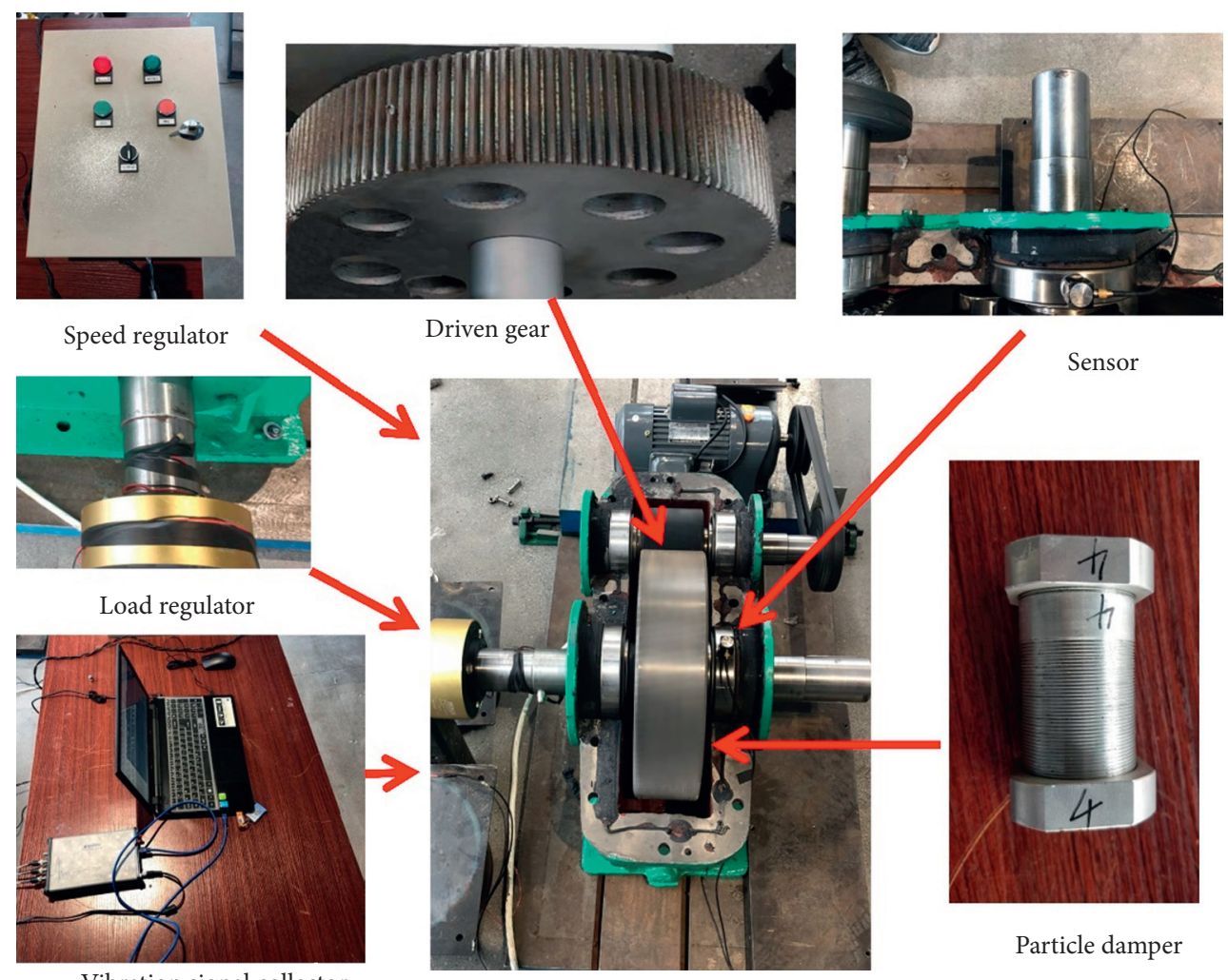

Vibration signal collector

Particle damper Figure 16: Gear transmission system test bench. 
TABLE 4: Root mean square value of acceleration.

\begin{tabular}{|c|c|c|c|c|c|c|c|}
\hline & None & $60 \%$ & $70 \%$ & $80 \%$ & $85 \%$ & $88 \%$ & $95 \%$ \\
\hline Test at $300 \mathrm{rpm}\left(\mathrm{m} / \mathrm{s}^{2}\right)$ & 9.63 & 8.036 & 7.241 & 6.604 & 6.13 & 5.7246 & 7.135 \\
\hline Test at $600 \mathrm{rpm}\left(\mathrm{m} / \mathrm{s}^{2}\right)$ & 10.904 & 9.88 & 9.032 & 8.041 & 7.278 & 6.691 & 7.904 \\
\hline Test at $1000 \mathrm{rpm}\left(\mathrm{m} / \mathrm{s}^{2}\right)$ & 12.735 & 11.105 & 10.235 & 9.24 & 8.374 & 7.716 & 8.884 \\
\hline
\end{tabular}

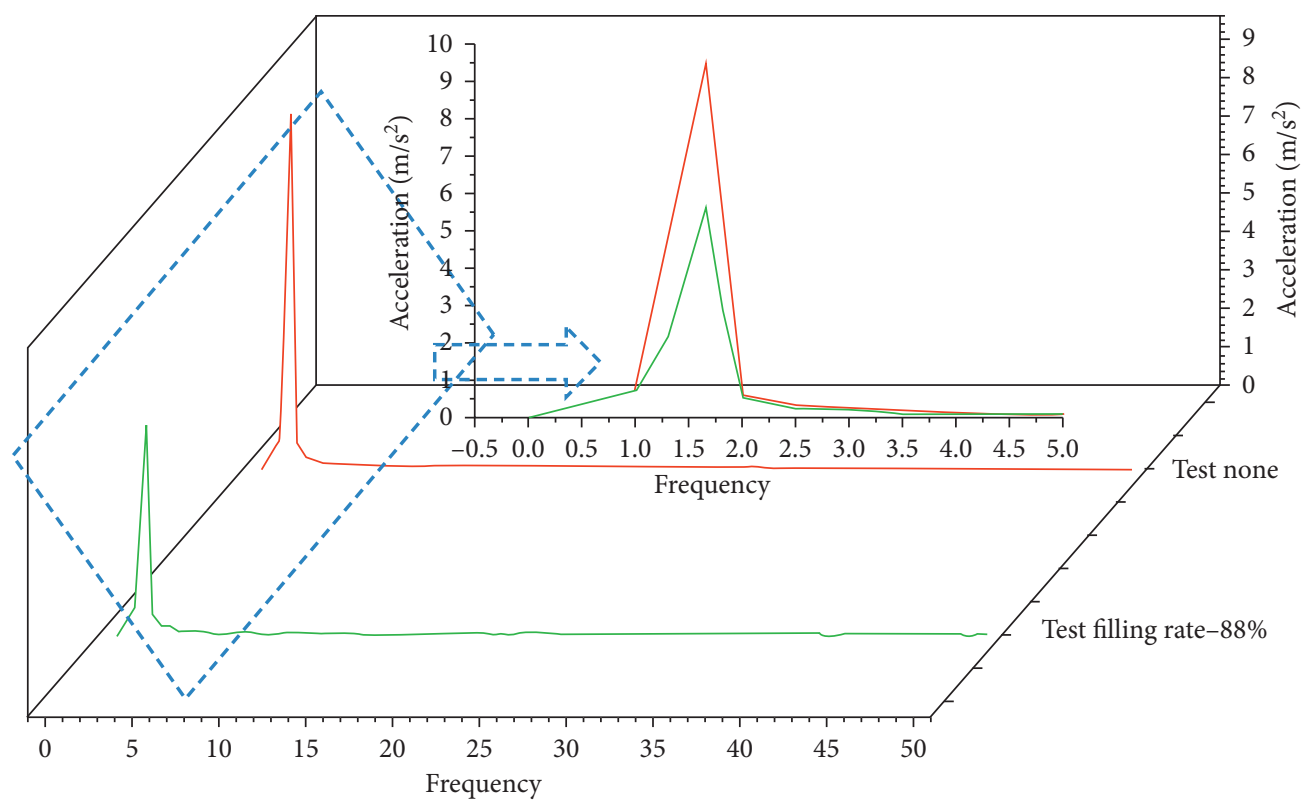

Figure 17: Acceleration spectrum.

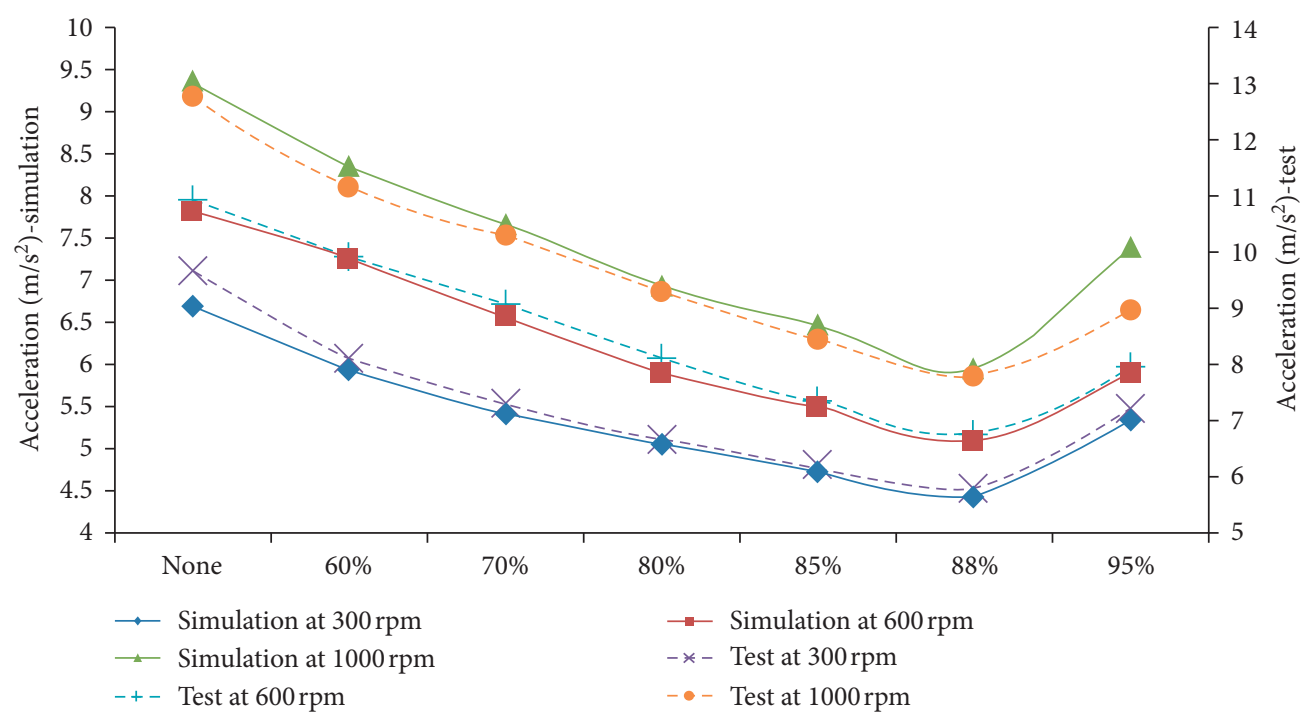

Figure 18: Comparison of test and simulation.

\section{Conclusion}

In this study, based on particle damping, a gear dynamic model was established by the coupling discrete element and finite element method. The vibration of the gear was reduced by installing the particle damper in the gear. By combining theoretical analysis with experimental verification, the influence of the filling rate of damping particles on the vibration reduction effect of the gearbox under different working conditions was studied.

In the process of gear coupling analysis, the contact force and position between the bulk medium and continuous medium were correctly transferred to the nodes corresponding to the finite element analysis model. The results 
showed that the filling rate of particles is an important factor affecting the damping effect of particle dampers.

With the increase of particle speed, particle damping effect is more obvious. However, with the increase of the centrifugal field, when the rotational speed exceeds a value, particle damping effect will decrease sharply due to the effect of strong centrifugal force. Therefore, for the gear test system in this study, when the filling rate of $2 \mathrm{~mm}$ tungsten particles is $88 \%$, the structural response is the smallest and the damping effect of the particle damper is the best. When the particle damping technology is applied to the gear, the rotational speed has great influence on the particle damper, so it is more necessary to combine the finite element and discrete element analysis.

\section{Data Availability}

The findings of the study cannot be shared at this time as the data also form part of an ongoing study.

\section{Conflicts of Interest}

The authors declare that they have no conflicts of interest.

\section{Acknowledgments}

This work was supported by National Natural Science Foundation of China (no. 51875490), Aeronautical Power Foundation of China (no. 6141B090562), Xiamen Science and Technology Planning Project (CN) (no. 3202Z20173021), Fundamental Research Funds for the Central Universities (CN) (no. 20720180063), and Science and Technology Project of Xiamen Construction Bureau (no. XJK2019-1-8).

\section{References}

[1] S. Wei, Q. Han, and F. Chu, "Review on research of uncertain dynamics for gear system," Journal of Mechanical Engineering, vol. 52, no. 1, pp. 1-19, 2016.

[2] S. Mo, T. Zhang, G.-G. Jin, X.-1. Cao, and H.-j. Gao, “Analytical investigation on load sharing characteristics of herringbone planetary gear train with flexible support and floating sun gear," Mechanism and Machine Theory, vol. 144, no. 2, pp. 1-27, 2020.

[3] S. Mo, Z. Yue, Z. Feng, L. Shi, Z. Zou, and H. Dang, "Analytical investigation on load-sharing characteristics for multipower face gear split flow system," Journal of Mechanical Engineering Science, vol. 234, no. 2, pp. 676-692, 2020.

[4] Y. Li, T. Chen, X. Wang, and K. Yu, "Non-linear dynamics of spur gear pair under external periodic excitation," Journal of Xi'an Jiaotong University, vol. 48, no. 1, pp. 101-105, 2014.

[5] J. Liu and L. Yuan, "Vibration analysis of a flexible gearbox system considering a local fault in the outer ring of the supported ball bearing," Journal of Vibration and Control, vol. 27, no. 9-10, pp. 1063-1076, 2020.

[6] P. Wang, Z. T. Su, S. Wang et al., "Research progress of graphene/rubber composite materials at home and abroad," Advanced Materials Industry, vol. 6, pp. 58-66, 2015.

[7] K. Feng, P. Borghesani, W. A. Smith et al., "Vibration-based updating of wear prediction for spur gears," Wear, vol. 426427, pp. 1410-1415, 2019.
[8] G. Liu and R. G. Parker, "Nonlinear dynamics of idler gear systems," Nonlinear Dynamics, vol. 53, no. 4, pp. 345-367, 2008.

[9] Z. Xu and R. Shao, "Forecast of sound pressure level of gear systems and fault diagnosis based on acoustics," Computer Measurement \& Control, vol. 17, no. 9, pp. 1688-1691, 2009.

[10] H. Li, C. Guo, S. Fang, and J. Ding, "Optimization design method for gearbox's vibration and noise reduction," Journal of Vibration and Shock, vol. 32, no. 17, pp. 150-154, 2013.

[11] S. C. Dragomir, M. Sinnott, and E. S. Semercigil, "Energy dissipation characteristics of the particle sloshing in a rotating cylinder," Journal of Sound and Vibration, vol. 331, no. 5, pp. 963-973, 2012.

[12] K. Li and A. P. Darby, "A buffered impact damper for multidegree-of-freedom structural control," Earthquake Engineering \& Structural Dynamics, vol. 37, no. 13, pp. 1491-1510, 2010.

[13] H. Jiang, C. Dong, and H. Ao, "Research on dynamic and static characteristics of metal rubber isolator used in aeroengine," Acta Aeronautica et Astronautica Sinica, vol. 25, no. 2, pp. 140-142, 2004.

[14] Y. Ma, Y. Li, G. Zhao, and J. Luo, "Research on the timedependent law of mechanical properties for the rubber isolation bearings based on thermal aging effect," Earthquake Engineering and Engineering Dynamics, vol. 1, no. 5, pp. 1844, 2017.

[15] B. M. Shah, D. Pillet, X. Bai, and M. K. Leon, "Construction and characterization of a particle-based thrust damping system," Journal of Sound and Vibration, vol. 126, no. 1, pp. 489-502, 2016.

[16] W. Xiao, Y. Huang, and H. Jiang, "Effect of powder material on vibration reduction of gear system in centrifugal field," Powder Technology, vol. 294, pp. 146-158, 2016.

[17] Z. Lu, X. Y. Chen, D. C. Zhang, and K. S. Dai, "Experimental and analytical study on the performance of particle tuned mass dampers under seismic excitation," Earthquake Engineering Structural Dynamics, vol. 46, no. 5, pp. 697-714, 2017.

[18] Z. Lu, K. Li, Y. T. Ouyang, and J. Z. Shan, "Performance-based optimal design of tuned impact damper for seismically excited nonlinear building," Engineering Structures, vol. 160, pp. 314-327, 2018.

[19] D. Guyomar and A. Badel, "Nonlinear semi-passive multimodal vibration damping: an efficient probabilistic approach," Journal of Sound and Vibration, vol. 294, pp. 249-268, 2006.

[20] S. J. Kim and S. K. Lee, "Experimental identification on a gear whine noise in the axle system of a passenger van," International Journal of Automotive Technology, vol. 8, pp. 75-82, 2007.

[21] Z. Lu, Z. X. Wang, S. F. Masri, and X. L. Lu, "Particle impact dampers: past, present, and future," Structural Control and Health Monitoring, vol. 25, no. 1, 2018.

[22] X. Lei, C. Wu, and P. Chen, "Optimizing parameter of particle damping based on Leidenfrost effect of particle flows," $\mathrm{Me}$ chanical Systems and Signal Processing, vol. 104, pp. 60-71, 2018.

[23] W. Xiao and W. Li, "Experiment for vibration suppression based on particle damping for gear transmission," Advances in Manufacturing Science and Engineering, vol. 945-949, pp. 703-706, 2014.

[24] W. Xiao, Z. Chen, T. Pan, and J. Li, "Research on the impact of surface properties of particle on damping effect in gear transmission under high speed and heavy load," Mechanical Systems and Signal Processing, vol. 98, pp. 1116-1131, 2018. 
[25] W. X. Shi, L. K. Wang, and Z. Lu, "Study on self-adjustable tuned mass damper with variable mass," Structural Control and Health Monitoring, vol. 25, no. 3, 2018.

[26] N. S. Weerasekara, M. S. Powell, P. W. Cleary et al., "The contribution of DEM to the science of comminution," Powder Technology, vol. 248, pp. 3-24, 2013.

[27] Y. Chung and J. Y. Ooi, "Linking of discrete element modelling with finite element analysis for analysing structures in contact with particulate solid," Powder Technology, vol. 217, pp. 107-120, 2012.

[28] J. Xu and Z. Tang, "Combined discrete/finite element multiscale numerical method and its application," Chinese Journal of Computational Physics, vol. 20, no. 6, pp. 477-482, 2003.

[29] B. Yao, Q. Chen, H. Xiang, and X. Gao, "Experimental and theoretical investigation on dynamic properties of tuned particle damper," International Journal of Mechanical Sciences, vol. 80, pp. 122-130, 2014.

[30] S. Xi, H. Cao, and X. Chen, "Dynamic modeling of spindle bearing system and vibration response investigation," $\mathrm{Me}$ chanical Systems and Signal Processing, vol. 114, pp. 486-511, 2019.

[31] W. Yu, C. K. Mechefske, and M. Timusk, "The dynamic coupling behavior of a cylindrical geared rotor system subjected to gear eccentricities," Mechanism and Machine Theory, vol. 107, pp. 105-122, 2017.

[32] Y. Shen, S. Yang, and X. Liu, "Nonlinear dynamics of a spur gear pair with time-varying stiffness and backlash based on incremental harmonic balance method," International Journal of Mechanical Sciences, vol. 48, pp. 1256-1263, 2006.

[33] S. Chen, J. Tang, and Y. Xie, "Analysis of nonlinear impact dynamic behavior for a gear pair system with time-varying stiffness and friction," Journal of Vibration and Shock, vol. 28, no. 4, pp. 76-80+204, 2009. 